\title{
Construction of a nursing solution to prevent and control urinary tract infection in the early stages of kidney transplantation
}

\author{
Zheng-Zheng Ma ${ }^{1 \# \wedge}$, Hu-Juan Yang ${ }^{1 \#}$, Xi Pan ${ }^{2}$, Ya-Dong Duan ${ }^{1}, \mathrm{Li} \mathrm{Li}^{1}$, Yan Xiao ${ }^{1}, \mathrm{Meng}-\mathrm{Yi}_{\mathrm{Cao}}{ }^{1}$, \\ Chun-Ya Qian ${ }^{1}$, Mei-E Niu ${ }^{3}$ \\ ${ }^{1}$ Department of Urology, the First Affiliated Hospital of Soochow University, Suzhou, China; ${ }^{2}$ Department of Neurology, the First Affiliated Hospital \\ of Soochow University, Suzhou, China; ${ }^{3}$ Department of Nursing, the First Affiliated Hospital of Soochow University, Suzhou, China \\ Contributions: (I) Conception and design: All authors; (II) Administrative support: ME Niu, CY Qian; (III) Provision of study materials or patients: \\ ZZ Ma, HJ Yang, CY Qian; (IV) Collection and assembly of data: ZZ Ma, HJ Yang; (V) Data analysis and interpretation: ZZ Ma, X Pan, YD Duan; (VI) \\ Manuscript writing: All authors; (VII) Final approval of manuscript: All authors. \\ \#These authors contributed equally to this work. \\ Correspondence to: Mei-E Niu. Department of Nursing, the First Affiliated Hospital of Soochow University, Suzhou 215006 , China. \\ Email: meniu@suda.edu.cn; Chun-Ya Qian. Department of Urology, the First Affiliated Hospital of Soochow University, Suzhou 215006, China. \\ Email: qianchunya01@163.com.
}

Background: To construct a nursing solution for the prevention and control of urinary tract infection (UTI) in the early stage after kidney transplantation, and to provide systematic and standardized nursing intervention measures for patients in the early stage after kidney transplantation.

Methods: The preliminary draft of intervention plan was formulated based on risk factor analysis research results of early UTI after kidney transplantation, combined with theoretical research, literature review, and research group meeting. The Delphi method was used to consult 15 experts for two rounds, and the entries were modified according to the opinions of the experts.

Results: After two rounds of consultation, the expert opinions tended to be consistent, and expert authority coefficient was 0.87 . The Kendall harmony coefficient of importance and feasibility indexes of the two rounds of consultation were $0.407,0.651$ and $0.545,0.686$, respectively, with statistically significant differences $(\mathrm{P}<0.001)$. The nursing solution consisted of eight first-level indexes and 35 second-level indexes. The eight first-level indexes included admission symptom evaluation, UTI monitoring, health education, sports intervention, nutrition intervention, ward management, risk factor prevention and nursing, and psychosocial intervention.

Conclusions: This study constructed a scientific and reliable nursing solution for the prevention and control of early UTI after kidney transplantation, which is hugely important for guiding clinical nursing work.

Keywords: Kidney transplantation; early urinary tract infection (early UTI); symptom management theory (SMT); Delphi technology; nursing

Submitted Sep 24, 2021. Accepted for publication Dec 01, 2021.

doi: $10.21037 /$ tau-21-926

View this article at: https://dx.doi.org/10.21037/tau-21-926

^ ORCID: 0000-0003-0253-6442. 


\section{Introduction}

Urinary tract infection (UTI) is the most common infectious complication after kidney transplantation (1), and the early postoperative period (i.e., $0-30$ days after surgery) is the peak stage of UTI $(2,3)$. Without timely prevention and control, UTIs can affect the function of kidney transplantation, increase the difficulty of treatment and the risk of death, and also result in enormous psychological and economic pressure to patients and their families (4). In addition, it is also a waste of transplanted organs, which are in short supply. The incidence of infection in kidney transplantation patients is mainly related to the exposure of potential pathogens in the environment, the application of immunosuppressive agents and the prevention of effective anti-infection. Therefore, the prevention and control of UTI after kidney transplantation is crucial.

Risk factors affecting the occurrence of UTI were analyzed, and early prevention and detection, as well as corresponding intervention measures were the key factors preventing and reducing the occurrence of UTI $(5,6)$. At present, the solutions based on the risk factors of UTI after kidney transplantation mostly involve drug intervention (7-10), and nursing intervention is rare. Multiple studies have confirmed that nursing intervention can reduce the incidence of UTI $(11,12)$, but there are few studies on nursing intervention, which only focus on specific patients after kidney transplantation. Delphi method is the expert consultation Method. The specific Method is to collect the opinions of the consulting experts on the problems to be consulted, and then conduct sorting, induction and statistical processing, and then feedback to the consulting experts anonymously again, and collect the opinions, focus and feedback again, until the experts' opinions tend to be consistent, the consultation is ended $(13,14)$. Therefore, based on the results of risk factor analysis of early UTI after kidney transplantation in the research group on prevention and control of UTI in the early stages of kidney transplantation (2), combined with theoretical research, literature review, and research group meeting, this study constructed a nursing solution for the prevention of UTI early after kidney transplantation using the Delphi method, which is reported below.

\section{Methods}

\section{Establishment of the research group}

The research team was composed of six specialists, with rich experience in kidney transplantation medical treatment and nursing, and two researchers. The expert members included two urologists, two nurses, one pharmacist, and one rehabilitation specialist [working years $(21.67 \pm 8.73)$ years; one undergraduate, three masters, two doctors; four senior titles, and two intermediate titles]. The expert members were mainly responsible for guiding the design of the project and the quality control of the research. Two project researchers, who were masters and bachelors of nursing, were mainly responsible for literature research, consultation questionnaire compilation, expert selection, questionnaire distribution and collection, and analysis and collation of the consultation results. The study conformed to the provisions of the Declaration of Helsinki (as revised in 2013), and was approved by the Institutional Review Board of The First Affiliated Hospital of Soochow University [No. (2021)208].

\section{Formation of the first draft of nursing solution for the prevention and control of early UTI after kidney transplantation}

\section{Theoretical study}

In this study, symptom management theory (SMT) was selected as the theoretical basis, which can be used to guide a single symptom and provide theoretical guidance for the development of effective symptom management strategies and related nursing research (15). Effective symptom management includes three basic elements: symptom experience, symptom management strategy, and management effect (16).

Based on the preliminary study of the research group, it was found that delayed recovery of renal function after renal transplantation was an independent risk factor for early UTI after renal transplantation (2). The intervention framework for early UTI prevention and control after kidney transplantation was established based on the experience of UTI, prevention and control strategies of UTI, and prevention and control effects of UTI (Figure 1).

\section{Literature review}

The keywords "kidney transplantation" and "urinary tract infection" were used to search the websites of domestic and overseas authoritative standard guidelines to check the nursing practice content involved in these guidelines. Using "kidney transplantation", "early period of postoperation", "urinary tract infection", and "nurse/nursing" as keywords, the research status and prevention/control nursing methods of early UTI after external kidney transplantation were 


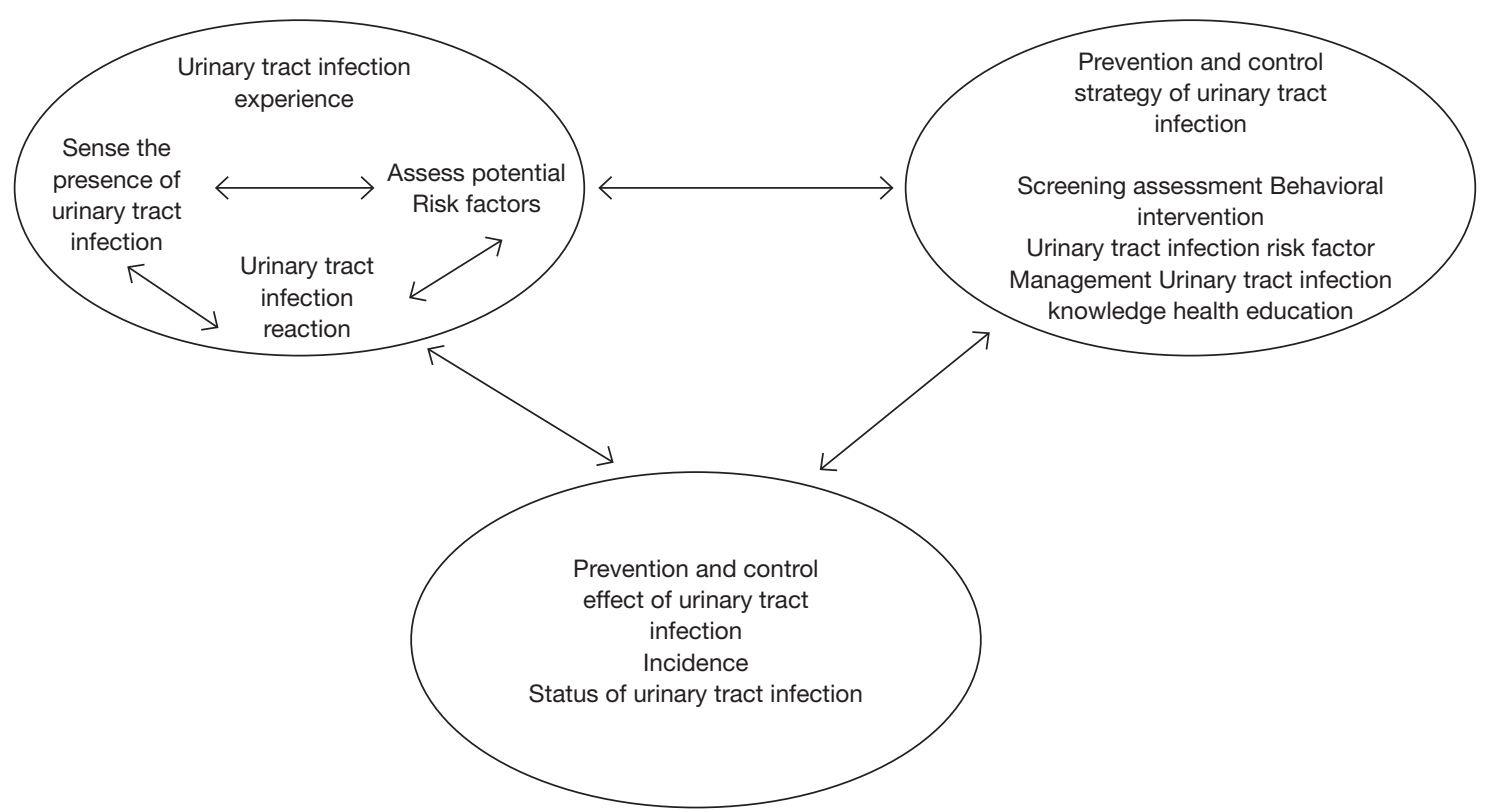

Figure 1 Framework for the prevention and control of early UTI after renal transplantation. UTI, urinary tract infection.

obtained from PubMed, Web of Science, CNKI, Wanfang, and other databases.

\section{Study group meeting}

We organized and held a research group meeting to determine the outline to be discussed according to the nursing plan of early UTI prevention and control after kidney transplantation obtained from the previous retrieval, including the suggestions, methods, and specific contents of the nursing plan of UTI prevention and control. According to the discussion outline, and combined with clinical experience, the research team members discussed and formed the first draft of the nursing plan for the prevention and control of UTI in the early stage after kidney transplantation.

\section{Expert consultation questionnaire formulation}

The expert consultation questionnaire consisted of three parts: (I) research introduction, which was a brief explanation of the research background, purpose, significance, and filling method; (II) self-evaluation table, which included the general information of experts, experts' familiarity with the content of the questionnaire, and the basis for judgment, and (III) consultation table of expert opinions on nursing solutions for the prevention and control of early UTI after kidney transplantation, which involved sorting of the contents of the solution according to first- and second-level indexes. Each index contained scoring columns of "importance" and "feasibility". The Likert five-level scoring method was adopted for scoring, and 1-5 points were assigned, from "not important or not feasible" to "very important or very feasible". In addition, each index had a column of suggestions for modification, so that experts could modify, supplement, or delete each index.

\section{Selection of consultants}

Fifteen experts were conveniently selected from Shanghai, Nanjing, and Suzhou. The selection criteria were as follows: (I) those engaged in the medical or nursing work of kidney transplantation; (II) doctor degree or above in the medical field, bachelor degree or above in nursing field; (III) working years $>10$ years; (IV) senior title in medical field, intermediate or above title in nursing field. All of the experts provided informed consent and volunteered to participate in the study.

\section{Implementation of expert consultation}

From January to February 2021, the researchers distributed and collected questionnaires through field investigation. After the first round of expert consultation, the researchers modified the indexes according to the screening criteria and 
expert opinions to form the second round questionnaire. The screening criteria for indicators were that both the mean importance score and the mean operability score were $>3.50$, and that the coefficient of variation was $<0.25$. The interval and filling period of each round of consultation were 2 weeks. After the second round of consultation, the expert opinions showed a good centralized trend, so the consultation was stopped, and the scheme was modified and improved according to the results of the second round of consultation.

\section{Statistical methods}

The IBM SPSS Statistics 23 was used for data statistical analysis in this study. The measurement data of experts' general data were expressed as mean \pm standard deviation, and the counting data were expressed as frequency and percentage. The importance and feasibility of each index were evaluated by the mean, standard deviation, and coefficient of variation. The expert positive coefficient was expressed through the questionnaire recovery rate. The degree of expert authority was expressed by the expert authority coefficient. The degree of expert opinion coordination was expressed by the coefficient of variation and the Kendall harmony coefficient. $\mathrm{P}<0.05$ was considered statistically significant.

\section{Results}

\section{General information of experts}

The same experts were consulted in the two rounds of this study. The 15 selected experts were $33-57(42.60 \pm 6.13)$ years old; $8(53.33 \%)$ were in clinical nursing and $7(46.67 \%)$ were in clinical healthcare; there were $6(40.00 \%)$ with working years ranging from 10 to 20 years, and $9(60.00 \%)$ with working years $\geq 20$ years. Also, there were 7 undergraduates $(46.67 \%), 1$ master $(0.07 \%)$, and 7 doctors (46.67\%). Additionally, there were 4 intermediate titles (26.67\%) and 11 senior titles (73.33\%).

\section{Expert positive coefficient, degree of authority, and degree of coordination of opinions}

The expert positive coefficient was expressed by the questionnaire recovery rate and the number of experts' comments. Fifteen questionnaires were sent out in the two rounds of consultation, and 15 were recovered, with an effective recovery rate of $100 \%$, which was $>80 \%$, illustrating the high enthusiasm of experts. In the first round, 7 (46.67\%) experts submitted 11 opinions orally or in writing, and in the second round, 2 (13.33\%) experts submitted two opinions orally, indicating that the experts participated well.

The degree of expert authority was expressed by the expert authority coefficient. This coefficient was the arithmetic mean of the experts' familiarity with the index and the basis of their judgment. Generally, an expert authority coefficient $>0.70$ indicates that experts have a good authority. The expert judgment basis of the two rounds of consultation was 0.89 , the expert familiarity was 0.85 , and the expert authority coefficient was 0.87 , indicating that the expert authority was high.

The degree of coordination of expert opinions was expressed by the Kendall harmony coefficient and the variation coefficient. The Kendall harmony coefficient was $0-1$, with greater values indicating a better degree of coordination. The Kendall harmony coefficient of the two rounds of consultation in this study is shown in Table 1. In the first round of expert consultation, the coefficient of variation of importance score was $0-0.178$, and the coefficient of variation of feasibility score was $0-0.213$. The coefficient of variation of importance score of the second round of expert consultation was $0-0.178$, and the coefficient of variation of feasibility score was $0-0.229$. The importance score, feasibility score, and variation coefficient of each index in this study are shown in Table 2.

\section{Expert consultation results}

According to the inclusion criteria, the indexes were not deleted in the two rounds of expert consultation. According to the opinions and suggestions of experts, the indexes were modified after discussion by the research group. After the first round of consultation, "symptom monitoring" was changed to "urinary tract infection monitoring" and "prevention and control of risk factors" was changed to "prevention and care of risk factors" in the first-level indicators. In the secondary indexes, "ask the patient about the history of UTI" was changed to "ask the patient about the history of UTI and analyze the causes"; "daily UTI screening evaluation and risk factor screening for patients" was changed to "daily UTI screening evaluation and risk factor screening for patients at 09:00"; "screening patients for women or delayed recovery of renal function after kidney transplantation should be evaluated twice a day" 
Table 1 Kendall harmony coefficient of two rounds of expert consultation

\begin{tabular}{|c|c|c|c|c|c|c|}
\hline Items & \multicolumn{3}{|c|}{ Importance } & \multicolumn{3}{|c|}{ Feasibility } \\
\hline \multicolumn{7}{|l|}{ First round } \\
\hline First-level index & 0.151 & 15.842 & 0.027 & 0.150 & 15.711 & 0.028 \\
\hline Total & 0.407 & 250.602 & $<0.001$ & 0.651 & 400.076 & $<0.001$ \\
\hline \multicolumn{7}{|l|}{ Second round } \\
\hline First-level index & 0.161 & 16.897 & 0.018 & 0.231 & 24.294 & 0.001 \\
\hline Second-level index & 0.588 & 300.028 & $<0.001$ & 0.695 & 354.400 & $<0.001$ \\
\hline
\end{tabular}

was changed to "screening patients for women or delayed recovery of renal function after kidney transplantation should be evaluated every 8 hours". In "if suspected UTI is found, please immediately consult a doctor for diagnosis", specific measures were added, including "timely replacement of urinary catheters" and "urine collection for bacterial culture and drug sensitivity test"; "preventive measures for early UTI after kidney transplantation" was changed to "preventive measures for risk factors of early UTI after kidney transplantation"; "increase fluid intake as the patient's condition allows. Ensure the urine volume was $50 \mathrm{~mL}$ per hour to maintain the patency of urination, prevent tube blockage, and maintain the patency of the urinary duct system" was changed to "increase fluid intake as the patient's condition allows. Ensure an average urine volume of $50 \mathrm{~mL}$ per hour to maintain the patency of urination, prevent tube blockage, and maintain the patency of the urinary duct system"; "perineum disinfection with $0.5 \%$ povidone iodine once per shift" was changed to "perineum disinfection with $0.5 \%$ povidone iodine three times daily (09:00, 15:00, 21:00)".

After the second round of consultation, "psychological intervention" was changed to "psychosocial intervention" in the first-level index, and "avoid bladder irrigation" was changed to "minimize bladder irrigation, and if necessary, refer to the color of the drainage fluid and the corresponding irrigation rate on the post-TURP CBI rate adjustment card (17)" in the second-level index. The results of the second round of expert consultation are shown in Table 2. After two rounds of consultation, the expert opinions were basically consistent, and the research team decided to terminate the consultation. Finally, the nursing plan for the prevention and control included eight first-level indexes and 35 second-level indexes.

\section{Discussion}

\section{The nursing plan of early UTI prevention and control after kidney transplantation is scientific}

Studies $(5,6)$ have shown that the incidence of symptoms can be prevented by treatment and nursing according to risk factors. The factors that may affect early UTI after kidney transplantation were analyzed by the research group based on literature review as well as the actual situation of our transplant center. The results showed that delayed recovery of renal function in women and after renal transplantation was an independent risk factor for early UTI after renal transplantation $(2,18)$. In addition, this study closely focused on symptom management strategy, one of the three basic elements of SMT, in order to develop an early UTI prevention and control nursing solution after kidney transplantation.

Symptom management strategies are used to prevent and control negative health status through biomedical, professional management, and self-care strategies (14). In previous studies, some scholars used SMT as a research framework to prevent and control the occurrence of delirium in patients with end-stage cancer, and managed the symptoms through early screening, early prevention, monitoring and evaluation, thus achieving certain effects (19). The prevention and control plan established 
Table 2 Results of the second round of expert consultation

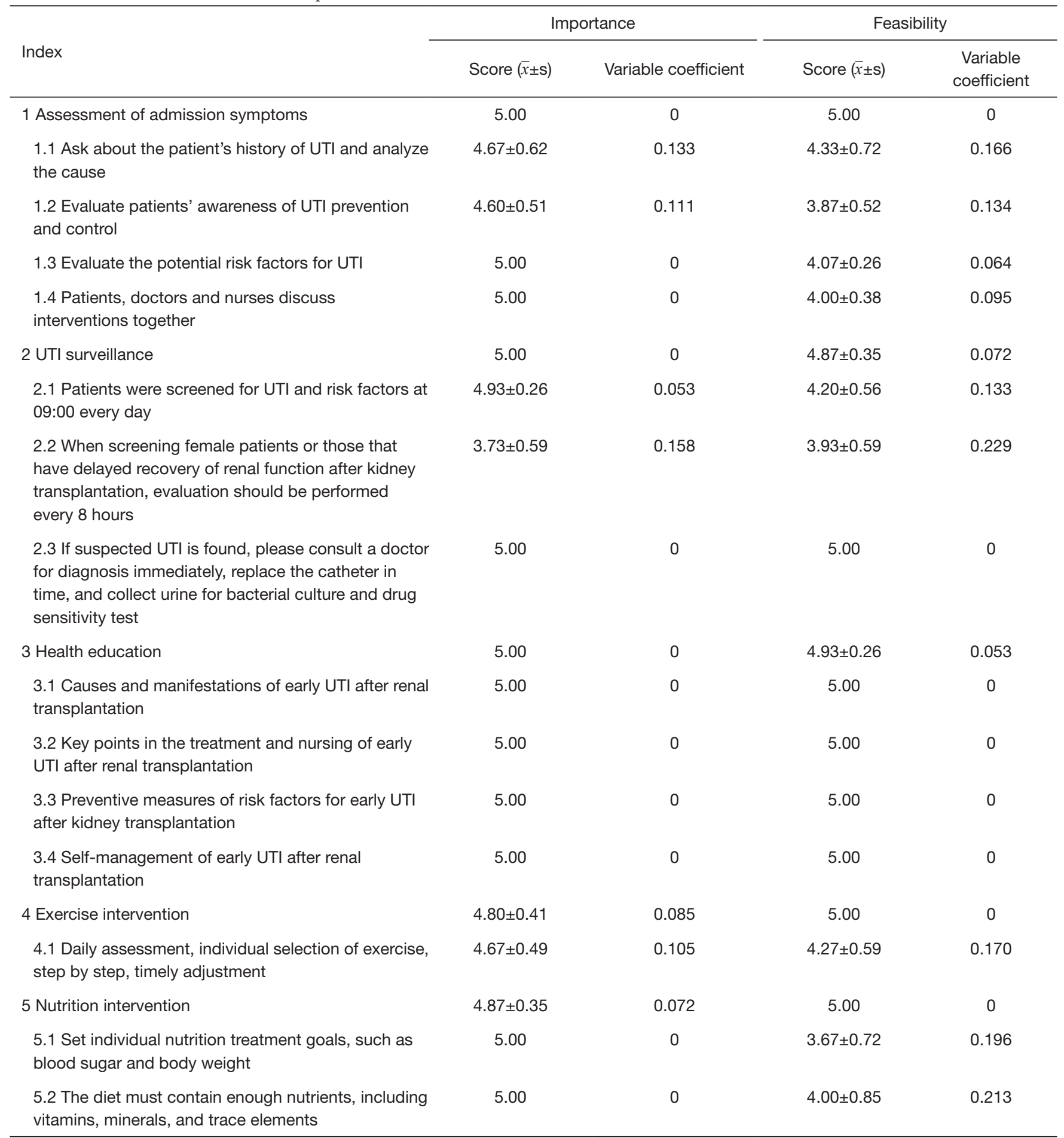

Table 2 (continued) 
Table 2 (continued)

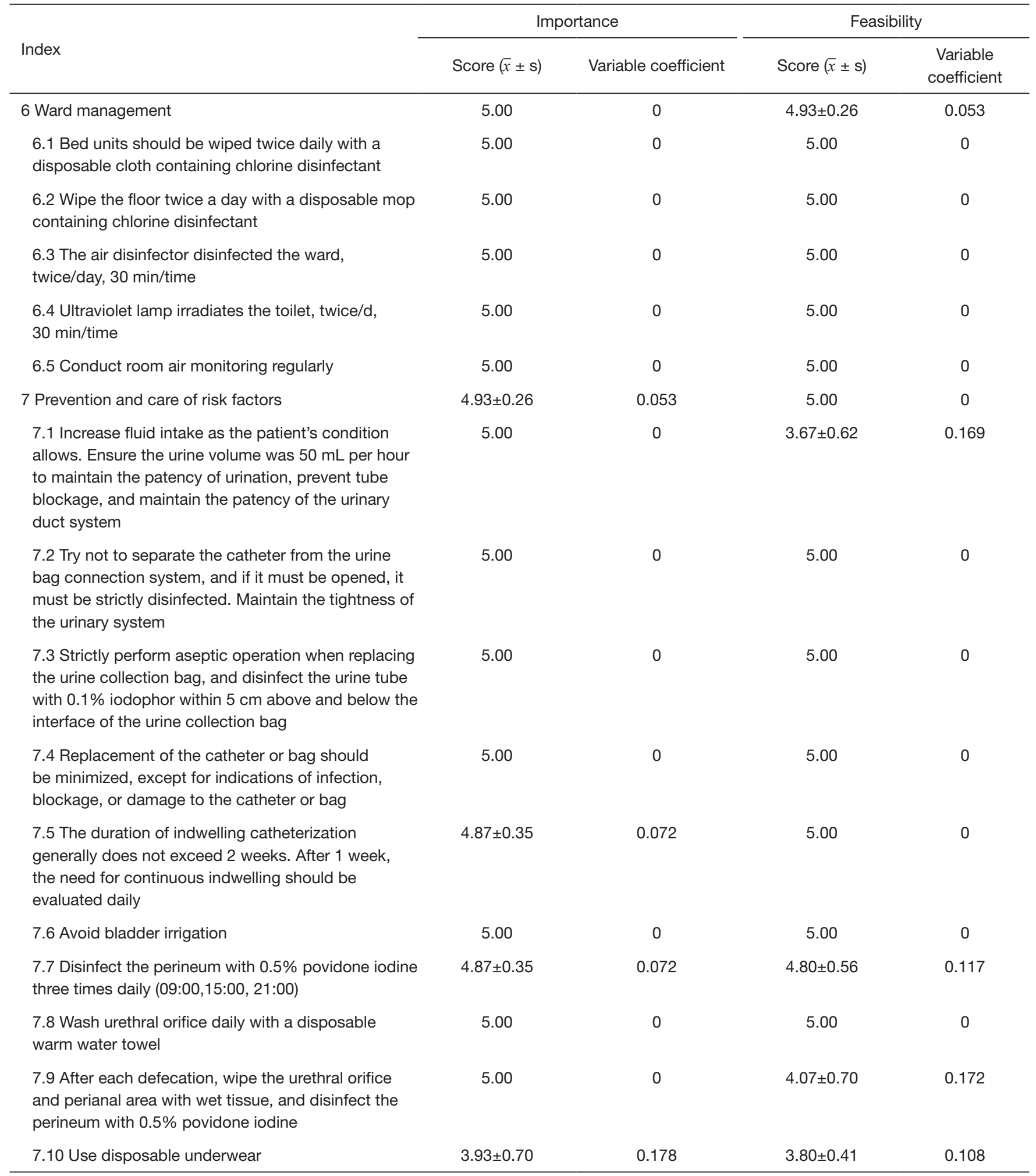

Table 2 (continued) 
Table 2 (continued)

\begin{tabular}{|c|c|c|c|c|}
\hline Index & \multicolumn{2}{|c|}{ Importance } & \multicolumn{2}{|c|}{ Feasibility } \\
\hline $\begin{array}{l}7.12 \text { Urine culture, sputum culture, and wound } \\
\text { drainage fluid culture collected daily. Early antibiotics } \\
\text { should be determined according to patient test } \\
\text { results, and the use of antibiotics should be } \\
\text { standardized to prevent the occurrence of multiple } \\
\text { drug resistant bacteria }\end{array}$ & 5.00 & 0 & 5.00 & 0 \\
\hline 8 Mental intervention & $4.73 \pm 0.46$ & 0.097 & $4.60 \pm 0.51$ & 0.111 \\
\hline 8.3 Encourage communication with fellow patients & $4.67 \pm 0.49$ & 0.105 & $4.87 \pm 0.35$ & 0.072 \\
\hline $\begin{array}{l}8.4 \text { Introduce common psychological intervention } \\
\text { methods, such as negative emotion intervention } \\
\text { therapy and meditation }\end{array}$ & $4.87 \pm 0.35$ & 0.072 & $4.47 \pm 0.64$ & 0.143 \\
\hline
\end{tabular}

in this study was based on the research results of risk factor analysis of early UTI after renal transplantation of our research group (2) and SMT, coupled with literature analysis, research group meeting, and the Delphi expert consultation method. This solution not only has sufficient a theoretical basis, but also contains rich practical experience. The established prevention and control solution included evaluation of admission symptoms, monitoring of UTI, health education, sports intervention, nutrition intervention, ward management, prevention and care of risk factors, and psychosocial intervention, which had a certain scientific nature.

\section{The nursing solution of early UTI prevention and control after kidney transplantation is reliable}

At present, there is no clear nursing solution for the prevention and control of early UTI after kidney transplantation, and there are few intervention studies on the nursing of UTI after kidney transplantation. Therefore, it is necessary to extensively solicit and listen to the opinions of authoritative experts in the field of kidney transplantation. In this study, 15 medical and nursing experts from three grade 3, class A hospitals in China were selected, all of whom had rich experience in clinical treatment or nursing of kidney transplantation and had a good understanding of UTI. The average working years of the consulting experts in this study was 20 years, and the experts have the better representation. The positive coefficients of the consultation experts in the two rounds of this study were $100 \%$, and a total of nine experts put forward constructive opinions in the two rounds, indicating that the experts were highly motivated and the research content attracted their attention. The average authority degree of experts in the two rounds of consultation in this study was 0.87 (which was $>0.70$ ), indicating that the authority of experts consulted in this study was high, the prediction accuracy was high, and the results were credible. In this scheme, the Likert grade 5 scoring method was adopted to screen the indexes. The mean scores of importance and operability of indexes were both $>3.50$, and the coefficient of variation was $<0.25$, ensuring the reliability of data collection and analysis results (20).

\section{Constructing a nursing plan for prevention and control of early UTI after kidney transplantation is of great significance}

Kidney transplantation is currently the best means to 
prolong the life of patients with end-stage renal disease $(21,22)$. In 2019, 40,608 people donated organs after death, and 153,863 organ transplants were performed, an increase of 4.80 percent from 2018, with an average of 17.5 transplants performed every hour. However, there are more than 1.3 million patients waiting for organ transplantation worldwide, and the current annual number of operations can only meet approximately $10 \%$ of the transplant needs (23). Therefore, the effective use of scarce transplant organ resources, as well as improving the survival rate and reducing the incidence of complications after kidney transplantation is particularly critical. In recent years, although great progress has been made in the treatment and nursing after kidney transplantation, UTI, the most common postoperative infectious complication, still has an incidence of $10-86 \%$ (24-26), and its overall prevention and control status is not optimistic. The outline of the "Healthy China 2030" plan points out that health services should be optimized to promote early recovery from diseases (27). Therefore, this study constructed a targeted nursing intervention solution to provide a reference for nurses to timely and effectively prevent and control UTI in early patients after kidney transplantation, improve the quality of nursing services, and promote the rehabilitation of patients with kidney transplantation.

\section{Conclusions}

A nursing solution for the prevention and control of early UTI after kidney transplantation was constructed in this study, which fully considered the actual work of clinical nursing, and was of high scientific nature and reliability. The next step will be to carry out the clinical application of this protocol to verify its effectiveness.

\section{Acknowledgments}

The authors gratefully thank Prof. Yuhua Huang, Dr. Linkun Hu and Dr. Yuxin Lin (Department of Urology, the First Affiliated Hospital of Soochow University, Suzhou, China) for their kind help in quality control and data collection.

Funding: This study was funded by "Zhoushi" Nursing Research Project of the First Affiliated Hospital of Soochow University (2021) (No. HLYJ-Z-202102) and Suzhou 32nd Batch of Science and Technology Development Plan (Medical and Health Technology Innovation) (2021) (No. SKJY2021065).

\section{Footnote}

Data Sharing Statement: Available at https://dx.doi. org/10.21037/tau-21-926

Conflicts of Interest: All authors have completed the ICMJE uniform disclosure form (available at https://dx.doi. org/10.21037/tau-21-926). The authors have no conflicts of interest to declare.

Ethical Statement: The authors are accountable for all aspects of the work in ensuring that questions related to the accuracy or integrity of any part of the work are appropriately investigated and resolved. All of the experts provided informed consent and volunteered to participate in the study. The study conformed to the provisions of the Declaration of Helsinki (as revised in 2013) and was approved by the Institutional Review Board of The First Affiliated Hospital of Soochow University [No. (2021)208].

Open Access Statement: This is an Open Access article distributed in accordance with the Creative Commons Attribution-NonCommercial-NoDerivs 4.0 International License (CC BY-NC-ND 4.0), which permits the noncommercial replication and distribution of the article with the strict proviso that no changes or edits are made and the original work is properly cited (including links to both the formal publication through the relevant DOI and the license). See: https://creativecommons.org/licenses/by-nc-nd/4.0/.

\section{References}

1. Korth J, Kukalla J, Rath PM, et al. Increased resistance of gram-negative urinary pathogens after kidney transplantation. BMC Nephrol 2017;18:164.

2. Ma ZZ, Li L, Han YX, et al. Analysis of risk factors for early urinary tract infection after kidney transplantation. Transl Androl Urol 2020;9:2211-7.

3. Clark JY, Raman JD. Urinary tract infection after radical cystectomy: a vexing problem despite prophylactic antibiotics. Transl Androl Urol 2019;8:S510-3.

4. Britt NS, Hagopian JC, Brennan DC, et al. Effects of recurrent urinary tract infections on graft and patient outcomes after kidney transplantation. Nephrol Dial Transplant 2017;32:1758-66.

5. Tawab KA, Gheith O, Al Otaibi T, et al. Recurrent Urinary Tract Infection Among Renal Transplant Recipients: Risk Factors and Long-Term Outcome. Exp Clin Transplant 
2017;15:157-63.

6. Rantz MJ, Alexander G, Galambos C, et al. Initiative to test a multidisciplinary model with advanced practice nurses to reduce avoidable hospitalizations among nursing facility residents. J Nurs Care Qual 2014;29:1-8.

7. Thompson ER, Hosgood SA, Nicholson ML, et al. Early versus late ureteric stent removal after kidney transplantation. Cochrane Database Syst Rev 2018;1:CD011455.

8. Kirnap M, Boyvat F, Torgay A, et al. Incidence of Urinary Complications With Double J Stents in Kidney Transplantation. Exp Clin Transplant 2019;17:148-52.

9. Ooms L, IJzermans J, Voor In 't Holt A, et al. Urinary Tract Infections After Kidney Transplantation: A Risk Factor Analysis of 417 Patients. Ann Transplant 2017;22:402-8.

10. Olenski S, Scuderi C, Choo A, et al. Urinary tract infections in renal transplant recipients at a quaternary care centre in Australia. BMC Nephrol 2019;20:479.

11. Viner S. Urinary tract infection knowledge of long-term care nursing staff: the effect of an educational intervention. Urologic Nursing 2020;40:7-10, 21.

12. Richards B, Sebastian B, Sullivan H, et al. Decreasing Catheter-Associated Urinary Tract Infections in the Neurological Intensive Care Unit: One Unit's Success. Crit Care Nurse 2017;37:42-8.

13. Dalkey N, Helmer O. An experimental application of the Delphi method to the use of experts. Management Science 1963;9:458-67.

14. Knippen K, Kuntzman L, Freeworth S. Patient Centered Approach to Postpartum Care after Gestational Diabetes: Delphi Method. J Acad Nutr Diet 2021;121:A45.

15. Smith MJ, Liehr PR. editors. Middle range theory for nursing. 4th ed. New York: Springer Publishing Company, 2018.

16. Dodd M, Janson S, Facione N, et al. Advancing the science of symptom management. J Adv Nurs 2001;33:668-76.

17. Ma ZZ, Han YX, Wang WZ, et al. The use of a homemade rate adjustment card in patients with continuous bladder irrigation after transurethral resection of the prostate. Transl Androl Urol 2020;9:2227-34.

18. Li L, Ma Z, Wang W. Influence of transitional care on the self-care ability of kidney transplant recipients after discharge. Ann Palliat Med 2020;9:1958-64.

19. Jablonski A, Wyatt GK. A model for identifying barriers to effective symptom management at the end of life. Journal of Hospice \& Palliative Nursing 2005;7:23-36.

20. Skulmoski GJ, Hartman FT, Krahn J. The Delphi method for graduate research. Journal of Information Technology Education: Research 2007;6:1-21.

21. Heemann U, Oberbauer R, Sprangers B, et al. Deceased donor kidney allocation schemes and international exchange. Curr Opin Organ Transplant 2020;25:66-73.

22. Kamburova EG, Hoitsma A, Claas FH, et al. Results and reflections from the PROfiling Consortium on Antibody Repertoire and Effector functions in kidney transplantation: A mini-review. HLA 2019;94:129-40.

23. GODT. Global transplantation activities of solid organs. 2019. Available online: http://www.transplantobservatory.org/

24. Bahrami A, Shams SF, Eidgahi ES, et al. Epidemiology of Infectious Complications in Renal Allograft Recipients in the First Year After Transplant. Exp Clin Transplant 2017;15:631-5.

25. Fiorentino M, Pesce F, Schena A, et al. Updates on urinary tract infections in kidney transplantation. J Nephrol 2019;32:751-61.

26. Rosado-Canto R, Parra-Avila I, Tejeda-Maldonado J, et al. Perioperative fosfomycin disodium prophylaxis against urinary tract infection in renal transplant recipients: a randomized clinical trial. Nephrol Dial Transplant 2020;35:1996-2003.

27. Li B. editor. Tutorial for outline of the healthy China 2030 plan. Singapore: Springer, 2020.

(English Language Editor: A. Kassem)
Cite this article as: Ma ZZ, Yang HJ, Pan X, Duan YD, Li L, Xiao Y, Cao MY, Qian CY, Niu ME. Construction of a nursing solution to prevent and control urinary tract infection in the early stages of kidney transplantation. Transl Androl Urol 2021;10(12):4392-4401. doi: 10.21037/tau-21-926 